\title{
Un caso infrecuente de aneurisma ateroesclerótico de la arteria temporal superficial ${ }^{*}$ Atherosclerotic aneurysm of the superficial temporal artery. Report of one case
}

\author{
Drs. RENÉ MORA O..$^{1,2}$, CRISTIÁN POZO U. ${ }^{1,3}$, CARLOS BARRÍA M. ${ }^{4}$, MARCELO A. BELTRÁN S. ${ }^{1,2}$, \\ RODRIGO BARRERA C. ${ }^{1,2}$, ALEJANDRA CLERICUS ${ }^{5}$, ALS. DANIELA NELSON F. ${ }^{2}$, DIEGO MORA C. ${ }^{6}$

\begin{abstract}
${ }^{1}$ Servicio de Cirugía Hospital de la Serena, ${ }^{2}$ Universidad Católica del Norte, Sede La Serena, ${ }^{3}$ Universidad Pedro de Valdivia, Sede La Serena, ${ }^{4}$ Unidad de Patología Hospital de La Serena, ${ }^{5}$ Unidad de Anestesiología, Hospital de La Serena, ${ }^{6}$ Universidad del Desarrollo, Santiago Chile.
\end{abstract}

\begin{abstract}
RESUMEN
Introducción: Los aneurismas de la arteria temporal superficial (AATS) constituyen una rara condición infrecuentemente reportada, ya que se han publicado sólo 14 casos de AATS ateroescleróticos en la literatura mundial. Este hecho contrasta con los aproximadamente 400 casos de pseudoaneurismas traumáticos de arteria temporal superficial reportados tanto en publicaciones nacionales, como internacionales. Reporte de Caso: Paciente femenino de 66 años de edad con antecedente de hipertensión arterial en tratamiento hace 17 años. El motivo de consulta fue la presencia de una masa pulsátil indolora en la región temporal derecha de 14 meses de evolución. Se estudió con ecografía Doppler vascular, la cual describe un aneurisma fusiforme de $2 \mathrm{~cm}$ de diámetro y $3 \mathrm{~cm}$ de longitud de la arteria temporal superficial. La cirugía consiste en la disección simple del aneurisma y la resección completa de la lesión con ligadura de los extremos proximales y distales de la arteria comprometida. El estudio histopatológico informa compromiso de las tres capas de la pared vascular en el área del aneurisma; también se observa la presencia de vasos adventiciales ateroescleróticos y engrosamiento ateroesclerótico de la íntima. La tinción de Van Gieson para fibras elásticas demuestra la integridad de la lámina elástica interna arterial. Conclusiones: Aún cuando los aneurismas verdaderos de la arteria temporal superficial son muy raros, su diagnóstico, estudio y tratamiento son relativamente simples y se encuentran actualmente bien establecidos.
\end{abstract}

PALABRAS CLAVE: Aneurisma, arteria temporal superficial, ateroesclerosis.

\begin{abstract}
Superficial temporal artery aneurysms (STAA) are an uncommon condition. Only 14 atherosclerotic STAA had been reported. This contrasts with almost 400 cases of superficial temporal artery traumatic
\end{abstract}

\footnotetext{
* Recibido el 18 de Enero de 2008 y aceptado para publicación el 6 de Marzo del 2008.

Correspondencia: Dr. René Mora O.

Balmaceda 916. La Serena, Chile

E-mail: moravascular@gmail.com
} 
pseudoaneurysms reported. We report a 66 years old female with hypertension treated for 17 years. She consulted for a painless pulsatile mass on the right temporal region lasting 14 months. A Doppler vascular ultrasound, described a superficial temporal artery fusiform aneurysm of $2 \mathrm{~cm}$ diameter and $3 \mathrm{~cm}$ length. The surgical exploration revealed the presence of the aneurysm compromising the superficial temporal artery. Simple dissection and resection of the lesion, ligating all the involved vascular branches was performed. The histopathological study reported involvement of all three layers of the vascular wall over the aneurysmatic area, also reported the presence of atherosclerotic adventitial vessels and atherosclerotic thickening of the intima. The Van Gieson stain for elastic fibers demonstrated integrity of the internal elastic layer.

\section{KEY WORDS: Temporal artery, aneurysm, atherosclerosis.}

\section{INTRODUCCIÓN}

Los aneurismas de la arteria temporal superficial (AATS) constituyen una rara condición infrecuentemente reportada en la literatura ${ }^{1-4}$. Cuando se presentan, los AATS se asocian a la presencia de aneurismas en otras arterias, al síndrome de Marfan y a la arteritis de células gigantes ${ }^{5-7}$. La ateroesclerosis es una causa infrecuente de AATS ${ }^{1,2}$; se han publicado sólo 14 casos de AATS ateroescleróticos en la literatura mundial, incluyendo el presente caso ${ }^{2}$. Este hecho contrasta con los aproximadamente 400 casos de pseudoaneurismas traumáticos de arteria temporal superficial reportados tanto en publicaciones nacionales ${ }^{8}$, como internacionales ${ }^{9-19}$. En el presente reporte discutimos un caso de AATS verdadero recientemente tratado en nuestra institución.

\section{REPORTE DE CASO}

Paciente femenino de 66 años de edad. Refiere como único antecedente de importancia hipertensión arterial, en tratamiento hace 17 años. El motivo de consulta fue la presencia de una masa pulsátil indolora en la región temporal derecha de aproximadamente 14 meses de evolución (Figura 1).

Se estudió con una ecografía Doppler vascular, la cual describe un aneurisma fusiforme de $2 \mathrm{~cm}$ de diámetro y $3 \mathrm{~cm}$ de longitud de la arteria temporal superficial. Otros estudios radiológicos solicitados para completar el estudio preoperatorio incluyeron una radiografía de tórax y ecografía abdominal buscando la presencia de otros aneurismas en los grandes vasos arteriales del tórax y abdomen, y estos estudios fueron normales. La exploración quirúrgica de la región temporal revela la presencia del aneurisma en la arteria temporal superficial (Figura 2).

Se realiza la disección simple del aneurisma y resección completa de la lesión con ligadura de los extremos proximales y distales de la arteria com- prometida. El estudio histopatológico informa compromiso de las tres capas de la pared vascular (intima, media y adventicia) y, en el área del aneurisma, también se observa la presencia de vasos adventiciales ateroescleróticos (Figura 3 ) y engrosamiento ateroesclerótico de la íntima. La tinción de Van Gieson para fibras elásticas demuestra la integridad de la lámina elástica interna de la arteria confirmando la presencia de un aneurisma verdadero de arteria temporal superficial (Figura 4). En el postoperatorio la paciente evoluciona sin incidentes y es dada de alta en buenas condiciones. Al seguimiento 8 meses después de la cirugía se encuentra asintomática.

\section{DISCUSIÓN}

Thomas Bartholin describió en 1740 por primera vez un aneurisma de arteria temporal, el cual fue secundario a un traumatismo cerrado de la región temporal por lo que actualmente es considerado como un pseudoaneurisma ${ }^{1,3,8,14}$.

El primer caso de aneurisma ateroesclerótico de arteria temporal histológicamente confirmado fue reportado por Martin y Shoemaker en $1955^{20}$. Desde entonces muy pocos casos de aneurismas verdaderos de arteria temporal superficial de etiología ateroesclerótica han sido reportados, algunos de ellos asociados a aneurismas en otras arterias $^{1-4}$. Los aneurismas pueden clasificarse como aneurismas verdaderos, aneurismas falsos o pseudoaneurismas y aneurismas disecantes ${ }^{10-13,15}$.

La diferencia entre un aneurisma verdadero y un pseudoaneurisma se encuentra en el compromiso de las capas histológicas de la pared vascular: cuando están intactas las tres capas se confirma la presencia de un aneurisma verdadero ${ }^{1-3,8,10}$, cuando se encuentra una solución de continuidad en una de las capas de la pared vascular se habla de un pseudoaneurisma ${ }^{1,3,10}$. Histopatológicamente la lámina elástica debe estar intacta para clasificar el aneurisma como verdadero o pseudoaneurisma. En nuestro caso la lámina elástica, así como la 


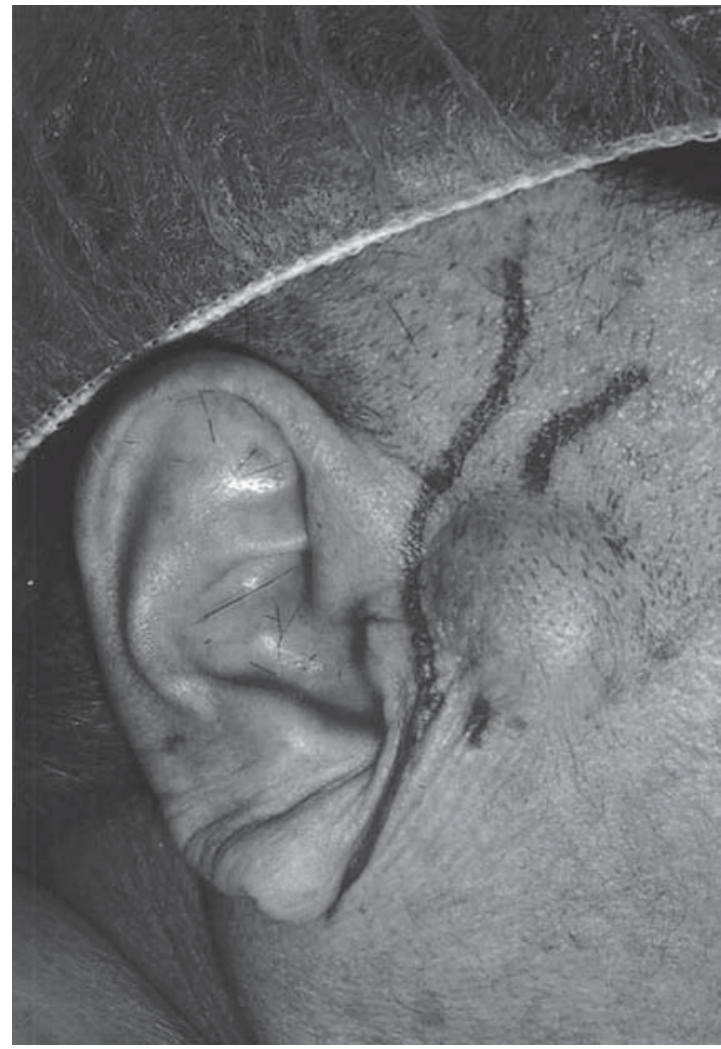

Figura 1. Aneurisma temporal antes de la operación.

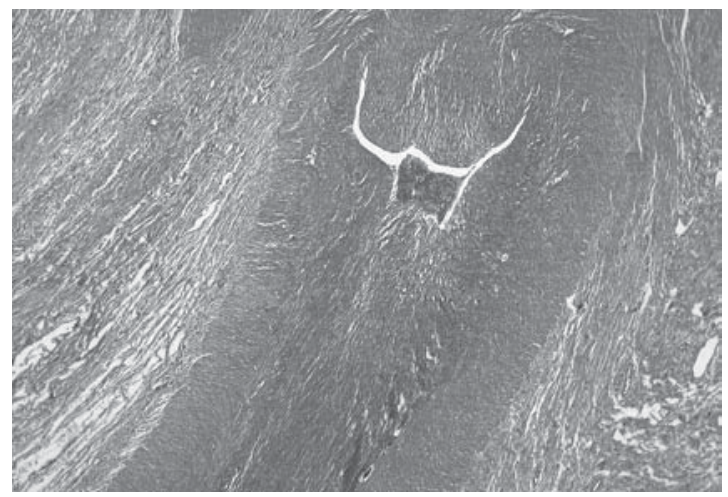

Figura 3. Vasos adventiciales ateroescleróticos (HE $40 \mathrm{x})$.

íntima, media y adventicia de la pared vascular se encontraban intactas, por lo que se consideró que este aneurisma se produjo debido una debilidad localizada de la pared vascular inducida por la placa de ateroma y asociada a la progresión de la aterosclerosis en nuestra paciente. El mecanismo fisiopatológico que explica el desarrollo de los AATS

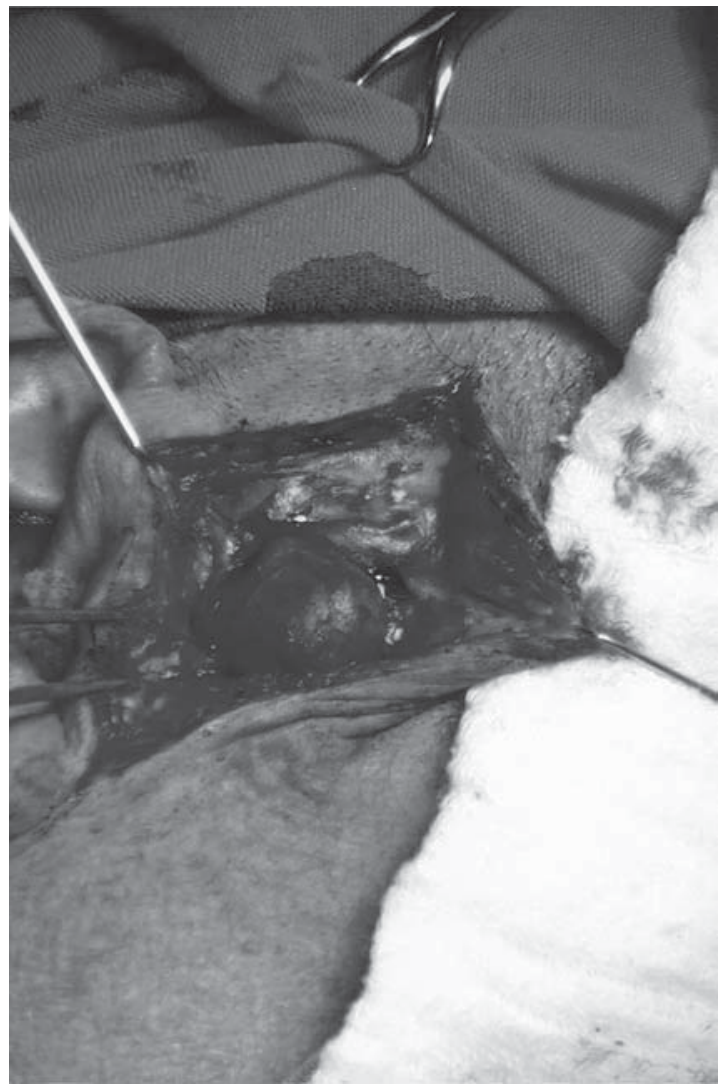

Figura 2. Imagen intraoperatoria del aneurisma.

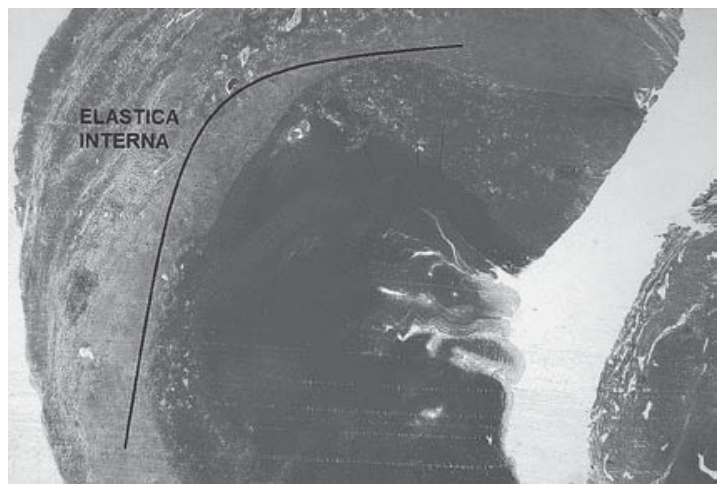

Figura 4. Lámina elástica interna indemne (Van Gieson $10 \mathrm{x})$.

es desconocido ${ }^{2}$, probablemente involucra cambios ateroescleróticos de la pared vascular y las consecuentes alteraciones hemodinámicas.

El diagnóstico clínico de los AATS no es difícil: la presencia de una masa pulsátil en la región temporal o preauricular, el soplo audible a la auscultación, el frémito y la fácil depresión a la palpa- 
ción son suficientes para proponer un diagnóstico presuntivo $0^{2,3,12-15}$. Los síntomas habitualmente descritos asociados a los AATS incluyen cefalea que puede ser intratable, hematoma y equimosis local cuando se rompen y la presencia de soplo audible que puede ser intolerable para el paciente ${ }^{2,11,12}$. En el diagnóstico diferencial de los AATS se incluyen hematomas, quistes, lipomas, tumores parotídeos, adenopatías, lesiones inflamatorias, tumores y aneurismas de la arteria meníngea media ${ }^{1,14}$. El diagnóstico diferencial más importante de los AATS es el pseudoaneurisma de arteria temporal superficial (PATS); el antecedente de trauma en la región temporal y preauricular permite sospechar la etiología de la masa pulsátil preauricular y presumir la presencia de un PATS ${ }^{8,10,13-15}$.

Ocasionalmente sin embargo, no existe el antecedente de trauma o el paciente no relaciona su problema con un trauma previo, por lo que el diagnóstico diferencial entre AATS y PATS se realiza mediante el examen histopatológico de la pieza quirúrgica. El AATS de nuestra paciente no presentó disrupción histológica de la lámina elástica ni de ninguna de las tres capas de la pared arterial, constituyendo un aneurisma verdadero, lesión extremadamente rara de acuerdo a la literatura actual. El estudio radiológico de estos casos incluye el uso de ecografía Doppler, que en nuestro caso resultó suficiente para confirmar el diagnóstico. Otros estudios descritos y utilizados incluyen la angiografía por sustracción digital, angiotomografía tridimensional y angioresonancia, las que no sólo confirman el diagnóstico sino que también descartan la presencia de otra patología asociada ${ }^{14,15,17}$. La angiografía selectiva es útil para el diagnóstico y también detecta la presencia de una fístula arteriovenosa; además puede ser eventualmente terapéutica si se realiza embolización del aneuris$m^{1-3,8,13,17}$. Nosotros utilizamos sólo la ecografía Doppler para confirmar el diagnóstico que fue inicialmente clínico, creemos que el uso de los exámenes radiológicos en estos casos encuentra su utilidad en la confirmación del diagnóstico clínico y en el estudio del paciente buscando patología asociada.

La cirugía está indicada para el alivio de los síntomas, corrección del defecto cosmético y prevención de las complicaciones del aneurisma, la que incluyen la expansión y ruptura. Adicionalmente, el aumento del tamaño del aneurisma puede producir compresión de arterias y nervios adyacentes causando sensación de adormecimiento y parestesia facial ${ }^{1,12,13}$. Actualmente el tratamiento de elección de estos aneurismas es la ligadura quirúrgica de todas las ramas arteriales comprometidas y su resección bajo anestesia local o ge- neral|-3,8-13, 15,18. Otras opciones terapéuticas disponibles en algunos centros son la embolización del aneurisma, la cual ha sido reportada con éxito $^{2,3,9,15,17,19}$ y el uso de derivados de trombina ${ }^{16}$. Nuestra paciente fue operada bajo anestesia general, cursó un postoperatorio sin incidentes y se encuentra asintomática, confirmando así los buenos resultados reportados con el tratamiento quirúrgico de estos aneurismas.

\section{CONCLUSIÓN}

Aún cuando los aneurismas verdaderos de la arteria temporal superficial son muy raros, su diagnóstico, estudio y tratamiento son relativamente simples y se encuentran actualmente bien establecidos. Creemos que la baja incidencia de estos aneurismas se debe en parte a que muchas veces se los considera simples curiosidades y no son reportados en la literatura.

\section{REFERENCIAS}

1. Uchido N, Sakuma M. Atherosclerotic superficial artery aneurysm: Report of a case. Surg Today 1999; 29: 575-578.

2. Endo T, Mori K, Maeda M. Multiple atherosclerotic fusiform aneurysms of the superficial temporal artery. Neurol Med Chir 2000; 40: 321-323.

3. Blanco EC, Benito JJ, Benito F, Gómez JL, del Cañizo Álvarez A, Antúnez P. Aneurisma no pulsátil de la arteria temporal superficial: A propósito de un caso. Acta Otorrinolaringol Esp 2003; 54: 388391.

4. Riaz AA, Ismail M, Sheik N, Ahmed N, Atkin G, Richman $\mathrm{P}$, et al. Spontaneously arising superficial temporal artery aneurysm: A report of two cases and review of the literature. Ann R Coll Surg Engl 2004; 86: 38-40.

5. Harrison RJ, Harrison CV, Kopelman H. Giant-cell arteritis with aneurysms: Effects of hormone therapy. Br Med J 1955; 31: 1593-1595.

6. Ross L, Truman J. Atypical bilateral temporal artery disease. Calif Med 1967; 107: 172-175.

7. Hu Z, Yang Q, Yang L, Li J, Tang J, Zhang $H$. Cerebral infarction due to giant-cell arteritis: Three case reports. Angiology 2004; 55: 227-231.

8. Álvarez HM, Bravo MM; Arancibia NL, Chacón GH. Pseudoaneurisma de la arteria temporal: Reporte de un caso y revisión de la literatura. Rev Chil Cir 2006; 58: 469-472.

9. Peick AL, Nichols WK, Curtis JJ, Silver D. Aneurysms and pseudoaneurysms of the superficial temporal artery caused by trauma. J Vasc Surg 1988; 8: 606-610.

10. Choo MJ, Yoo IS, Song HK. A traumatic pseudoaneurysm of the superficial temporal artery. Yonsei Med J 1988; 39: 180-183. 
11. Pipinos II, Dossa CD, Reddy DJ. Superficial temporal artery aneurysms. J Vasc Surg 1998; 27: 374377.

12. Magloire FD, Reyes RC, Magloire PM. Aneurisma de la arteria temporal superficial: Reporte de un caso. Rev Mex Angiol 1999; 27: 41-44.

13. Weller CB, Reeder RC. Traumatic pseudoaneurysm of the superficial temporal artery: Two cases. J Am Osteopath Assoc 2001; 101: 284-287.

14. Walker MT, Liu BP, Salehi SA, Babve S, Batjer HH. Superficial temporal artery pseudoaneurysm: Diagnosis and preoperative planning with CT angiography. Am J Neuroradiol 2003; 24: 147-150.

15. Isaacson G, Kochan PS, Kochan JP. Pseudoaneurysms of the superficial temporal artery: Treatment options. Laryngoscope 2004; 114: 10001004.

16. Bobinski L, Boström S, Hillman J, Theodorsson A.
Postoperative pseudoaneurysm of the superficial temporal artery (S.T.A.) treated with Thrombostatâ (thrombin glue) injection. Acta Neurochir 2004; 146: 1039-1041.

17. Higashino T, Kawashima M, Mannoji H. Three-dimensional computed tomography angiography for the investigation of superficial artery pseudoaneurysms: Two case reports. Neurol Med Chir 2005; 45: 152-155.

18. Cavalcante FSBL, Villas CVM, Alvarenga ACG. Traumatic pseudoaneurysm of the superficial temporal artery. Arq Neuropsiquiatr 2005; 63: 859-861.

19. Hong JT, Lee SW, Ihn YK, Son BC, Sung JH, Kim IS, et al. Traumatic pseudoaneurysm of the superficial temporal artery treated by endovascular coil embolization. Surg Neurol 2006; 66: 86-88.

20. Martin WL, Shoemaker WC. Temporal artery aneurysm. Am J Surg 1955; 28: 696-702. 